Invited Paper

\title{
Bifurcation analyses of nonlinear dynamical systems: From theory to numerical computations
}

\author{
Kunichika Tsumoto $^{1}$, Tetsushi Ueta ${ }^{2 a)}$, Tetsuya Yoshinaga ${ }^{3}$, \\ and Hiroshi Kawakami 4 \\ ${ }^{1}$ Division of Molecular and Cellular Pharmacology, Graduate school of \\ Medicine, Osaka University \\ 2-1 Yamada-oka, Suita, Japan \\ ${ }^{2}$ Center for Administration of Information Technology, The University of \\ Tokushima \\ 2-1, Minami-josanjima, Tokushima, Japan \\ ${ }^{3}$ Institute of Health Biosciences, The University of Tokushima \\ 3-18-15, Kuramoto, Tokushima, Japan \\ 4 Japan Science and Technology Agency, \\ 4-1-8, Honmachi, Kawaguchi, Saitama, Japan \\ a) ueta@tokushima-u.ac.jp
}

Received April 3, 2012; Revised June 4, 2012; Published October 1, 2012

\begin{abstract}
In this paper, we explain how to compute bifurcation parameter values of periodic solutions for non-autonomous nonlinear differential equations. Although various approaches and tools are available for solving this problem nowadays, we have devised a very simple method composed only of basic computational algorithms appearing in textbooks for beginner's, i.e., Newton's method and the Runge-Kutta method. We formulate the bifurcation problem as a boundary value problem and use Newton's method as a solver consistently. All derivatives required in each iteration are obtained by solving variational equations about the state and the parameter. Thanks to the quadratic convergence ability of Newton's method, accurate results can be quickly and effectively obtained without using any sophisticated mathematical library or software. If a discontinuous periodic force is applied to the system, we can use the same strategy to solve the bifurcation problem. The key point of this method is deriving a differentiable composite map from the various information about the problem such as the location of sections, the periodicity, the Poincaré mapping, etc.
\end{abstract}

Key Words: nonlinear dynamical system, bifurcation, boundary value problem, variational equation, Newton's method 


\section{Introduction}

Many biological, social, and engineering phenomena are dynamic, and their phenomena can be modeled as dynamical systems [1]. Most dynamical systems are described by ordinary differential equations or difference equations. In general, these systems are nonlinear and include many parameters. Small changes in the values of their parameters may have large effects on the behaviors of the system. Determining a way to analyze such a dynamical system is an important problem. So far, a large number of methods of analyzing nonlinear dynamical systems have been proposed. As is well known, the solution to most nonlinear dynamical systems cannot be obtained analytically. This means we must conduct numerous simulations using the different fixed sets of parameter values and initial conditions. However, such simulations only give information about one stable solution at a time, and they tend to take a long time to reach a solution.

The topological properties of the solutions to a dynamical system may change when a parameter of the system changes slightly. This phenomenon is called bifurcation. Examples of bifurcation phenomena include a transition from a stable equilibrium state to an oscillating motion or from a regular oscillation to a chaotic state. Bifurcation analysis, which is the investigation of bifurcations depending on the system parameters, is a way to gain deep insights into the fundamental properties of dynamical systems. Furthermore, bifurcation analysis enables us to identify the range of a parameter over which a system behaves stably, the total behavior of the solution in the large, and the transition mechanisms of the dynamic responses.

A set of parameter values that cause bifurcations is called a bifurcation set, and a graph of these sets is called a bifurcation diagram. Two or three decades ago, finding a bifurcation value was very troublesome task; in fact, many researchers took a process of trial and error to trace a bifurcation set manually. These days however, computational tools, for instance, AUTO and MATCONT [2-4], can be used to perform bifurcation analyses. On the other hand, Kawakami developed a bifurcation analysis tool thirty years ago [5], and since then, he and his co-workers have improved it [6-16] to the point that the current version can compute any bifurcation set within a few hours. Indeed, before this development, researchers were often forced to devote up to a whole year to make one bifurcation diagram! Indeed, although computers have helped us to achieve our goals, the study of algorithms remains a very important topic.

Assume that you have a nonlinear problem and want to compute its bifurcation diagram. Although AUTO is a useful and quick tool for this task, its results are especially difficult for beginners to understand.

In this article, we consider a dynamical system described as an ordinary differential equation or difference equation, and explain in detail the shooting algorithm to trace the bifurcation sets appearing in the system, in particular, a non-autonomous system with periodic forcing. Unlike the terse style of many technical papers, we shall make a more text-book style of presentation; i.e., we will carefully and explicitly explain our method, and we will not shy away from helpful pedagogical redundancies.

This article is organized as follows. Section 2 briefly describes the basic theory of bifurcation in nonlinear dynamical systems. Section 3 summarizes the numerical computation for the bifurcation analysis. It also illustrates our method by using it to analyze a typical non-autonomous system. Section 4 is the conclusion.

\section{Basic theory of bifurcation analysis}

Let us consider the following autonomous system:

$$
\frac{d x}{d t}=f_{a}(x, \lambda)
$$

and its forced system or the following non-autonomous system:

$$
\frac{d x}{d t}=f(t, x, \lambda)
$$

where $t \in R$ denotes time, $x \in R^{n}$ is a vector consisting of state variables, and $\lambda \in R^{\ell}$ denotes system parameters. Now, let us assume that $f_{a}$ in Eq. (1) and $f$ in Eq. (2) are sufficiently differentiable, i.e., 
$C^{\infty}$-class. In addition, let us assume that the function $f$ is periodic in $\tau_{p}$ so that $f\left(t+\tau_{p}, x, \lambda\right)=$ $f(t, x, \lambda)$, for all $t$. We shall also assume that a solution to Eq. (1) or (2) with the initial condition $x=x_{0}$ at $t=t_{0}$ is described by $x(t)=\varphi\left(t, x_{0}\right)$, for all $t$, or $x(t)=\varphi\left(t, \lambda ; t_{0}, x_{0}\right)$, if necessary, to show the parameters and the initial state at the initial time $t_{0}$ explicitly.

\subsection{Equilibrium point and variational equation in autonomous system}

Now let us briefly review the case of autonomous systems obeying Eq. (1). By imposing the conditions:

$$
f_{a}(x, \lambda)=0,
$$

one can compute the locations of the equilibrium points in the system. A point $x^{*}$ satisfying Eq. (3) is an equilibrium point. The Jacobian matrix and its corresponding characteristic equation give enough information to determine the stability of each equilibrium point.

Assume that $x^{*}$ is an equilibrium point. The Taylor expansion of $f_{a}$ in Eq. (1) about the equilibrium point had by setting $x=x^{*}+\xi$ can be described as

$$
\frac{d\left(x^{*}+\xi\right)}{d t}=f_{a}\left(x^{*}+\xi\right)=f_{a}\left(x^{*}\right)+\left.\frac{\partial f_{a}(x)}{\partial x}\right|_{x=x^{*}} \xi+\cdots,
$$

where $\xi$ is a small perturbation. By subtracting this equation from Eq. (1), we obtain a variational equation with respect to $\xi$ :

$$
\frac{d \xi}{d t}=\left.\frac{\partial f_{a}(x)}{\partial x}\right|_{x=x^{*}} \xi=\frac{\partial f_{a}\left(x^{*}\right)}{\partial x} \xi \equiv J_{c} \xi
$$

Suppose that the eigenvalues $\mu_{i}, i=1,2, \ldots, n$, are the roots of the characteristic equation for this $n \times n$ Jacobian matrix $J_{c}$, and they determine the stability of the equilibrium point. For simplicity, we shall assume that all eigenvalues are real and distinct, and if the corresponding eigenvectors, $v_{i}$, for $i=1,2, \ldots, n$, can be obtained with a suitable scheme, the general solution of the variational equation Eq. (5) can be written as:

$$
\xi(t)=\sum_{i=1}^{n} c_{i} e^{\mu_{i} t} v_{i}
$$

where $c_{i}$ is a constant related to the initial values of the state variables in Eq. (5). Obviously, this general solution is stable if and only if all eigenvalues are negative. Note that this solution only describes the local behavior around the equilibrium point. Thus, the eigenvalues of the Jacobian matrix, $J_{c}$, express a stability index for the given equilibrium point in a continuous-time autonomous system.

Here, we call $x^{*}$ a hyperbolic equilibrium point, if the real parts of all eigenvalues are non-zero. A bifurcation occurs when an equilibrium point loses its hyperbolicity as a result of variation of the system parameters. In other words, bifurcation phenomena result from changes in the stability of the equilibrium point.

Typical bifurcations of the equilibrium point are as follows:

i The Hopf bifurcation: A couple of complex conjugate eigenvalues of the characteristic equation becomes purely imaginary numbers. An oscillatory solution appears as a result of changing the value of a parameter.

ii The saddle-node bifurcation: One of the eigenvalues is zero. A pair of equilibrium points appears when the value of a parameter is changed.

In this article, we shall omit the details of this equilibrium analysis and refer the reader to the literature [17-21].

Note that the existence and stability of equilibrium points has an effect on the dynamical behavior of the non-autonomous system of Eq. (2). If you add a very small periodic perturbation to an autonomous system, a periodic solution will appear around the equilibrium, and it will inherit the stability of the equilibrium. Mathematically speaking, adding a small perturbation to an autonomous system leads to the disappearance of all equilibrium points since $d x / d t \neq 0$ for all $t$. 


\subsection{Periodic solutions and fixed points in a non-autonomous system}

Next, let us discuss the stability of periodic solutions. The periodic solution to Eq. (2) can be qualitatively studied by using a Poincaré map.

\subsubsection{Poincaré mapping and fixed points}

Let us rewrite the solution to Eq. (2) starting from $x=x_{0}$ at $t=0$ as

$$
x(t)=\varphi\left(t, \lambda ; t_{0}, x_{0}\right) .
$$

Thus, the solution is also periodic in $\tau_{p}$ because of the periodicity of $f$ in Eq. (2), i.e., $\varphi(t+$ $\left.\tau_{p}, \lambda ; t_{0}, x_{0}\right)=\varphi\left(t, \lambda ; t_{0}, x_{0}\right)$. We can naturally define the corresponding map:

$$
\begin{aligned}
S_{\lambda}: R^{n} & \rightarrow R^{n} \\
x_{0} & \mapsto S_{\lambda}\left(x_{0}\right)=\varphi\left(t+\tau_{p}, \lambda ; t_{0}, x_{0}\right) .
\end{aligned}
$$

This map is actually a sampling of the orbit every $\tau_{p}$; thus, we get

$$
x_{0}, S_{\lambda}\left(x_{0}\right), S_{\lambda}^{2}\left(x_{0}\right), \cdots, S_{\lambda}^{m}\left(x_{0}\right), \cdots .
$$

We call this $S_{\lambda}$ the Poincaré mapping. A sequence (9) is obtained by neglecting information about continuous changes in $\varphi\left(t, \lambda ; t_{0}, x_{0}\right)$ during a period satisfying $t \in\left[k \tau_{p},(k+1) \tau_{p}\right)$, for $k=0,1,2, \ldots$ Indeed, this sort of reduction is a useful. Note also that the system of interest has been converted from a differential equation (2) into a difference equation:

$$
x^{(k+1)}=S_{\lambda}\left(x^{(k)}\right),
$$

for $k=0,1,2, \cdots$.

If an initial state, $x_{0} \in R^{n}$, satisfies the following relationship:

$$
x_{0}=S_{\lambda}\left(x_{0}\right),
$$

then this point is called a fixed point. Furthermore, if for some $m \neq 1$,

$$
x_{0}=S_{\lambda}^{m}\left(x_{0}\right),
$$

and if all $S_{\lambda}^{k}\left(x_{0}\right), k=0,1, \ldots, m-1$, are different each other, $x_{0}$ is called an m-periodic point. Thereby, we can obtain a one-to-one correspondence between the periodic solution of Eq. (2) and the fixed point of the Poincaré map $S_{\lambda}$. The case of an $m$-periodic point can also be studied simply by replacing $S_{\lambda}$ with $S_{\lambda}^{m}$, or the $m$ th iterate of $S_{\lambda}$, in Eq. (11). Therefore, for the sake of simplicity, we shall explain only the properties of a fixed point of $S_{\lambda}$.

In general, we cannot obtain the explicit form of $S_{\lambda}$, though a limited number of continuous dynamical systems do have one. However, it is easy enough to obtain the Poincaré mapping. For instance, we can obtain its Poincaré map by getting the values of the state variable every $\tau_{p}$ in the numerical simulation. Furthermore, in real electrical and electronic circuits, the voltage value of a periodic motion can be easily sampled with a sample-hold circuit by using trigger pulses synchronized with an external periodic force.

\subsubsection{Stability of a fixed point in difference equation}

Let us consider a stability index for difference equations. Equation (8) forms of itself a discrete dynamical system (difference equation) like as follows:

$$
z^{(k+1)}=W\left(z^{(k)}\right), \quad k=0,1,2, \ldots
$$

If one can find a fixed point $\zeta^{*}$ for the discrete dynamical system of Eq. (13),

$$
\zeta^{*}-W\left(\zeta^{*}\right)=0 .
$$


As a similar framework to that of Eq. (4), we can let $\eta^{(k)} \in R^{n}$ be a small perturbation away from the fixed point $\zeta^{*}$ satisfying Eq. (14). For a small $\varepsilon$, we can assume that $\left\|\eta^{(k)}\right\|<\varepsilon$, where $\|\cdot\|$ is the Euclidean norm. If the variation around the fixed point is defined as $z^{(k)}=\zeta^{*}+\eta^{(k)}$, the difference equation of Eq. (13) is

$$
z^{(k+1)}=\zeta^{*}+\eta^{(k+1)}=W\left(\zeta^{*}+\eta^{(k)}\right)
$$

A Taylor expansion gives

$$
\zeta^{*}+\eta^{(k+1)}=W\left(\zeta^{*}\right)+\left.\frac{\partial W}{\partial z}\right|_{z=\zeta^{*}} \eta^{(k)}+\cdots
$$

The following linear difference equation is obtained by neglecting high-order terms and subtracting this equation from Eq. (14):

$$
\eta^{(k+1)}=\left.\frac{\partial W}{\partial z}\right|_{z=\zeta^{*}} \eta^{(k)}=\frac{\partial W\left(\zeta^{*}\right)}{\partial z} \eta^{(k)} \equiv J_{d} \eta^{(k)}
$$

where $J_{d}$ is an $n \times n$ Jacobian matrix and its elements are derivatives of the solution with respect to the state variables.

The eigenvalue problem for a difference equation is formulated as:

$$
J_{d} v_{i}=\mu_{i} v_{i}
$$

for $i=1,2, \ldots, n$. In general, this equation has $n$-tuple non-zero vectors $v_{i}, i=1,2, \ldots, n$. We call $\mu_{i}$ and the corresponding $v_{i}$ a multiplier and eigenvector of the difference equation (17), respectively. The multipliers are computed from the following characteristic equation:

$$
\operatorname{det}\left(J_{d}-\mu_{i} I\right)=0
$$

and the eigenvectors are obtained directly from Eq. (18) with the specific $\mu_{i}$.

Here, let us give an intuitive interpretation of the multiplier. In particular, let us consider the case in which all multipliers of Eq. (19) are real and distinct. For any $i$, if an eigenvector $v_{i}$ is chosen as the initial point, the iterative dynamics of Eq. (17) can be expressed as an uncoupled difference equation:

$$
v_{i}^{(k+1)}=\mu_{i} v_{i}^{(k)}, \quad \text { with } \quad v_{i}^{(0)}=v_{i},
$$

and it generates the following vector sequence:

$$
\left\{v_{i}^{(0)}, v_{i}^{(1)}, v_{i}^{(2)}, \ldots, v_{i}^{(k)}, \ldots\right\}
$$

Since the movement is only in this eigenvector direction, we have

$$
v_{i}^{(1)}=\mu_{i} v_{i}^{(0)}=\mu_{i} v_{i}, \quad v_{i}^{(2)}=\mu_{i} v_{i}^{(1)}=\mu_{i}^{2} v_{i}, \quad \ldots, \quad v_{i}^{(k)}=\mu_{i}^{k} v_{i} .
$$

On the one hand, from linear systems theory, the solution at time $k$ to a linear difference equation (17) can be expressed as a linear combination of constants, multipliers, and eigenvectors:

$$
\begin{aligned}
\eta^{(k)} & =\sum_{i=1}^{n} c_{i} \mu_{i}^{k} v_{i} \\
& =c_{1} \mu_{1}^{k} v_{1}+c_{2} \mu_{2}^{k} v_{2}+\cdots+c_{n} \mu_{n}^{k} v_{n},
\end{aligned}
$$

Cf. Eq. (6). Notice that the iteration (22) multiplies each scalar $\mu_{i}$ by a certain eigenvector. The "multiplier" is sometimes referred to an "eigenvalue" in some literature, but "multiplier" expresses its actual feature in this case. To prevent an explosion in the magnitude of the vector $\eta^{(k)}$, all absolute values of $\mu_{i}$ should be less than unity.

As expected, the local stability (a topological property) of a fixed point is determined by the multipliers. Let us go back to the Poincaré mapping $S_{\lambda}$. It generates a sequence like: 


$$
x^{(1)}=S_{\lambda}\left(x_{0}\right), \quad x^{(2)}=S_{\lambda}\left(x^{(1)}\right), \quad \ldots, x^{(k+1)}=S_{\lambda}\left(x^{(k)}\right), \ldots
$$

From the linearity of the variational equation defined at Eq. (17), the characteristic equation for Eq. (19) can be written as:

$$
\operatorname{det}\left(\frac{\partial S_{\lambda}\left(x^{*}\right)}{\partial x}-\mu_{i} I\right)=0,
$$

where $x^{*}$ is a fixed point. If all the absolute values of the multipliers are different from unity, we call $x^{*}$ a hyperbolic fixed point of $S_{\lambda}$. We can topologically classify hyperbolic fixed points. For instance, Table I shows the topological classification of the hyperbolic fixed point in a two-dimensional discrete system. Readers should check the difference in the stability criterion between differential and difference equations. In addition, a clear classification of hyperbolic fixed points can be found in $[5,22,23]$.

Table I. Classification of fixed point.

\begin{tabular}{c|c|c}
\hline Name & Topological property & Condition \\
\hline sink & completely stable & $\left|\mu_{1}\right|<1,\left|\mu_{2}\right|<1$ \\
\hline source & completely unstable & $\left|\mu_{1}\right|>1,\left|\mu_{2}\right|>1$ \\
\hline saddle & direct-type unstable & $0<\mu_{1}<1<\mu_{2}$ \\
\hline saddle & inverse-type unstable & $\mu_{1}<-1<\mu_{2}<0$ \\
\hline
\end{tabular}

\subsubsection{Bifurcation of periodic solution}

The topological properties of fixed/periodic points might change as a result of changing the parameter value. Moreover, another attractor may appears when the topological properties change. We call this phenomenon bifurcation of a periodic solution.

There are three kinds of local bifurcation for periodic solutions (see Fig. 1).

i Tangent bifurcation (or saddle-node bifurcation of periodic solution, or fold bifurcation [17, 24]): This bifurcation causes a pair of a node and a saddle to disappear or emerge (Fig. 1(a)). In circuit experiments or numerical simulations, the fixed point makes a large leap in value when the parameter changes by one increment (this phenomenon is called a jump). Moreover, a fixed point that jumped in this way cannot return to the original position at the bifurcation parameter (This phenomenon is called hysteresis). At a particular parameter value $\lambda=\lambda^{*}$, one of the multipliers of the characteristic equation satisfies the condition $\mu_{i}=1$ (Fig. 1(d)).

ii Period-doubling bifurcation (or flip bifurcation $[17,25]$ ): This type of bifurcation occurs when a real characteristic multiplier passes through a point $(-1,0)$ in the complex plane, i.e., $\mu_{i}=-1$ (Fig. 1(d)). If an inverse-type saddle takes this value of the multiplier, its stability changes. As a side effect, two-periodic points are generated around the fixed point. In general, the period doubles. No its fixed point disappears.

iii Neimark-Sacker bifurcation (or a Hopf bifurcation in a discrete system $[17,25,26]$ ): Similar to the Hopf bifurcation for an equilibrium point, the fixed point becomes unstable, and an invariant closed curve, which corresponds to doubly periodic oscillation (quasi-periodic solution) of the original periodic non-autonomous system, may appear in the Poincaré map (Fig. 1(c)). This type of bifurcation occurs when a pair of multipliers $\mu_{i, i^{\prime}}$ pass transversely through points of the unit circle except for 0 and $\pi$, i.e., $\mu_{i, i^{\prime}}=\exp ( \pm j \theta)$, where $j=\sqrt{-1}$ and $\theta$ is the argument of the complex value.

\section{Method of numerical computation}

Now we will describe the methods for numerically computing bifurcations of a periodic solution.

If one has a mathematical model of Eq. (1) or (2) and it accurately describes the corresponding dynamical system, one may want to compute accurate locations of the equilibrium point or fixed point. Furthermore, one may want to obtain bifurcation sets in an arbitrary parameter space. 
(a) Tangent bifurcation

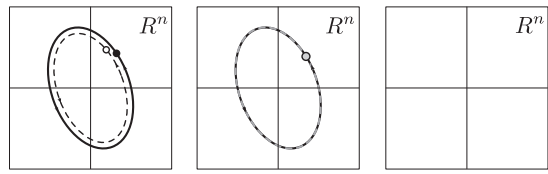

(b) Period-doubling bifurcation
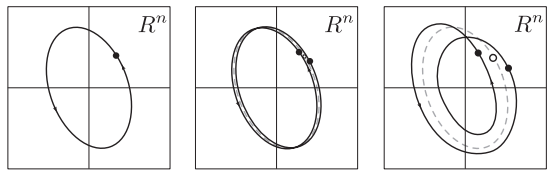

(c) Neimark-Sacker bifurcation
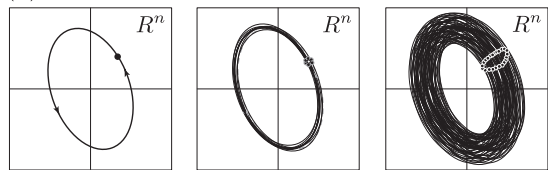

(d)

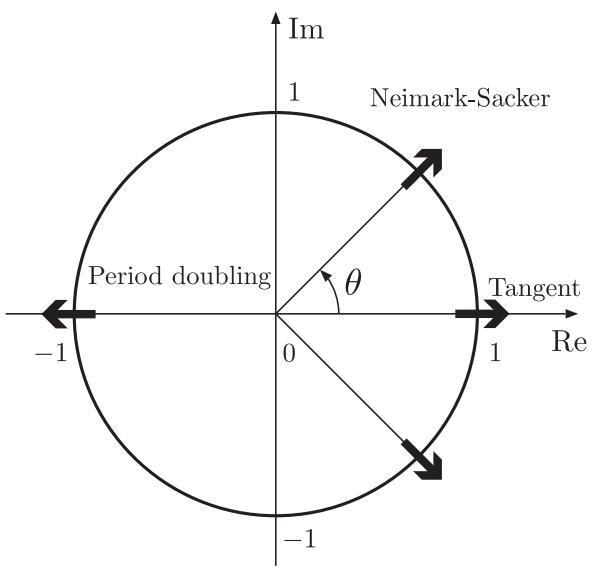

Fig. 1. Bifurcations of a periodic solution and the generic conditions. (a)-(c) In each phase plane, the solid and dashed line indicate stable and unstable periodic solutions, respectively. The black and white circles denote the stable and unstable fixed/periodic points, respectively. (d) The location of the multipliers on the complex plane.

We shall consistently use Newton's method to accomplish these tasks and calculate a solution satisfying $T\left(q^{*}\right)=0$ with sufficient accuracy, where $T(q)$ is an arbitrary nonlinear function in which where $q=\left(q_{0}, q_{1}, \ldots, q_{n}\right)^{\top}, T=\left(T_{1}, T_{2}, \ldots, T_{n}\right)^{\top}$, and $(\cdot)^{\top}$ represents the transpose operation. The Taylor expansion of the $k$ th approximation $q^{(k)}$ is

$$
T(q)=T\left(q^{(k)}\right)+D T\left(q^{(k)}\right)\left(q^{(k+1)}-q^{(k)}\right)+\cdots,
$$

where $D T\left(q^{(k)}\right)=\left(\partial T_{i} / \partial q_{j}\right)$ is the Jacobian matrix. A correction vector $h=q^{(k+1)}-q^{(k)}$ can be obtained by solving the following linear non-homogeneous equation:

$$
D T\left(q^{(k)}\right) h=-T\left(q^{(k)}\right)
$$

Since Newton's method generally has a quadratic convergence property except for cases such that the first derivatives at a solution are zero, a few iterations will give an accurate solution from a rough first-guess $q^{(k)}$.

\subsection{Tracking the fixed point}

Here, we perform Newton's method on Eq. (11):

$$
F\left(x_{0}\right):=x_{0}-S_{\lambda}\left(x_{0}\right)=0,
$$

where $x_{0}$ corresponds to a fixed point. In the following, the initial value in Eq. (2) is described as $x_{0}=\left(x_{01}, x_{02}, \ldots, x_{0 n}\right)^{\top}$. Furthermore, we have $x_{0}^{(k)}$ as a first-guess of the fixed point. Then the algorithm to obtain a $(k+1)$ th order approximation is as follows:

$$
\begin{aligned}
x_{0}^{(k+1)} & =x_{0}^{(k)}+h \\
D F\left(x_{0}^{(k)}\right) h & =-F\left(x_{0}^{(k)}\right)
\end{aligned}
$$

where $D F\left(x_{0}^{(k)}\right)$ is an $n \times n$ sized Jacobian matrix with respect to the initial value $x_{0}$ given by:

$$
D F\left(x_{0}^{(k)}\right)=I-\frac{\partial S_{\lambda}}{\partial x}\left(x_{0}^{(k)}\right)
$$

where $I$ is an $n \times n$ identity matrix, and $\partial S_{\lambda} / \partial x$ is the following matrix: 


$$
\frac{\partial S_{\lambda}}{\partial x}\left(x_{0}^{(k)}\right)=\left(\begin{array}{cccc}
\frac{\partial \varphi_{1}}{\partial x_{01}}\left(\tau_{p}, x_{0}^{(k)}\right) & \frac{\partial \varphi_{1}}{\partial x_{02}}\left(\tau_{p}, x_{0}^{(k)}\right) & \cdots & \frac{\partial \varphi_{1}}{\partial x_{0 n}}\left(\tau_{p}, x_{0}^{(k)}\right) \\
\frac{\partial \varphi_{2}}{\partial x_{01}}\left(\tau_{p}, x_{0}^{(k)}\right) & \ddots & & \vdots \\
\vdots & & \ddots & \frac{\partial \varphi_{n-1}}{\partial x_{0 n}}\left(\tau_{p}, x_{0}^{(k)}\right) \\
\frac{\partial \varphi_{n}}{\partial x_{01}}\left(\tau_{p}, x_{0}^{(k)}\right) & \cdots & \cdots & \frac{\partial \varphi_{n}}{\partial x_{0 n}}\left(\tau_{p}, x_{0}^{(k)}\right)
\end{array}\right)
$$

The second equation of Eq. (29) must be solved for $h$ by using a suitable method like the Gauss elimination. $F\left(x_{0}^{(k)}\right)$ is easily obtained from the original equation (28), but how does one obtain the actual elements in the Jacobian matrix (i.e., Eq. (31))?

As such, we should revisit Eq. (2):

$$
\frac{d x}{d t}=f(t, x, \lambda)
$$

where $x=\left(x_{1}, x_{2}, \ldots, x_{n}\right)^{\top}, f(t, x)=\left(f_{1}(t, x, \lambda), f_{2}(t, x, \lambda), \ldots, f_{n}(t, x, \lambda)\right)^{\top}$. We should also rewrite $\varphi=\left(\varphi_{1}, \varphi_{2}, \ldots, \varphi_{n}\right)^{\top}$. We had defined the solution starting from $x_{0}$ at $t=t_{0}$ as:

$$
x(t)=\varphi\left(t, \lambda ; t_{0}, x_{0}\right) \equiv \varphi\left(t, x_{0}\right) .
$$

Substituting the solution of this equation into Eq. (32), we get

$$
\frac{d \varphi\left(t, x_{0}\right)}{d t}=f\left(t, \varphi\left(t, x_{0}\right)\right) .
$$

Differentiating this equation by $x_{0}$ yields

$$
\frac{\partial}{\partial x_{0}}\left(\frac{d \varphi\left(t, x_{0}\right)}{d t}\right)=\frac{\partial}{\partial x_{0}}\left(f\left(t, \varphi\left(t, x_{0}\right)\right)\right) .
$$

The order of differentiation on the left-hand side is commutative, and the following equation is obtained from the right-hand side:

$$
\frac{d}{d t}\left(\frac{\partial \varphi\left(t, x_{0}\right)}{\partial x_{0}}\right)=\frac{\partial f\left(t, \varphi\left(t, x_{0}\right), \lambda\right)}{\partial x} \frac{\partial \varphi\left(t, x_{0}\right)}{\partial x_{0}} .
$$

This equation is of the following form:

$$
\frac{d X}{d t}=\frac{\partial f}{\partial x} X,
$$

where, $X=\partial \varphi / \partial x_{0}$ is the matrix solution of a variable coefficient linear differential equation. We call Eq. (36) a variational equation for Eq. (32). Obviously,

$$
\frac{\partial \varphi\left(0, x_{0}\right)}{\partial x_{0}}=I .
$$

Therefore, by setting Eq. (38) as the initial value, we can obtain all factors in Eq. (31) by numerically integrating Eq. (36) from $t=0$ to $\tau_{p}$. The Runge-Kutta method is a simple and powerful way of performing such an integration. Once this is completed, Newton's method is ready to perform. The fixed point $x_{0}$ is accurately located by iteration. To compute an $m$-periodic point, we can numerically integrate Eq. (36) for $0 \leq t<m \tau_{p}$ instead.

\subsection{Tracking bifurcation sets}

Now let us compute a bifurcation curve on a two-parameter plane. The algorithm is quite simple; it just adds one more condition to Eq. (28), that is, the characteristic equation of Eq. (25). Consider the following simultaneous equation:

$$
F_{B}:=\left[\begin{array}{c}
x_{0}-S_{\lambda}\left(x_{0}\right) \\
\operatorname{det}\left(D S_{\lambda}\left(x_{0}^{*}\right)-\mu^{*} I\right)
\end{array}\right]=0,
$$


where $D S_{\lambda}\left(x_{0}^{*}\right)$ denotes the derivative of the Poincaré map, $S_{\lambda}$, with respect to the initial value, $x_{0}$, i.e., $D S_{\lambda}\left(x_{0}^{*}\right)=\partial S_{\lambda}\left(x_{0}\right) /\left.\partial x_{0}\right|_{x_{0}=x_{0}^{*}}$. The first equation in Eq. (39) is the condition for a point on the map to be a fixed point, and the second equation is the condition by which the value of the characteristic equation must be zero at a specific value of $\mu^{*}$, e.g., $\mu=1$, for calculating the tangent bifurcation set.

We define $F_{B} \in R^{n+1}$ as:

$$
F_{B}\left(x_{0}, \lambda\right)=\left[g_{1}\left(x_{0}, \lambda\right), g_{2}\left(x_{0}, \lambda\right), \ldots, g_{n}\left(x_{0}, \lambda\right), \chi\left(x_{0}, \lambda, \mu^{*}\right)\right]^{\top}
$$

where $\chi\left(x_{0}, \lambda, \mu^{*}\right)$ is the characteristic equation. Newton's method can be used to solve Eq. (39). Now let us return to Eq. (29):

$$
\begin{aligned}
u^{(k+1)} & =u^{(k)}+h \\
D F_{B}\left(u^{(k)}\right) h & =-F_{B}\left(u^{(k)}\right)
\end{aligned}
$$

where $u=\left(x_{01}, x_{02}, \ldots, x_{0 n}, \lambda\right)^{\top}$. The Jacobian matrix is

$$
\begin{aligned}
& D F_{B}\left(u^{(k)}\right)=\left(\begin{array}{ccccc}
\frac{\partial g_{1}}{\partial x_{01}} & \frac{\partial g_{1}}{\partial x_{02}} & \cdots & \frac{\partial g_{1}}{\partial x_{0 n}} & \frac{\partial g_{1}}{\partial \lambda} \\
\frac{\partial g_{2}}{\partial x_{01}} & \ddots & & \vdots & \vdots \\
\vdots & & \ddots & \frac{\partial g_{n-1}}{\partial x_{0 n}} & \frac{\partial g_{n-1}}{\partial \lambda} \\
\frac{\partial g_{n}}{\partial x_{01}} & \cdots & \cdots & \frac{\partial g_{n}}{\partial x_{0 n}} & \frac{\partial g_{n}}{\partial \lambda} \\
\frac{\partial \chi}{\partial x_{01}} & \cdots & \cdots & \frac{\partial \chi}{\partial x_{0 n}} & \frac{\partial \chi}{\partial \lambda}
\end{array}\right) \\
& =\left(\begin{array}{ccccc}
1-\frac{\partial \varphi_{1}}{\partial x_{01}} & -\frac{\partial \varphi_{1}}{\partial x_{02}} & \cdots & -\frac{\partial \varphi_{1}}{\partial x_{0 n}} & -\frac{\partial \varphi_{1}}{\partial \lambda} \\
-\frac{\partial \varphi_{2}}{\partial x_{01}} & \ddots & & \vdots & \vdots \\
\vdots & & \ddots & -\frac{\partial \varphi_{n-1}}{\partial x_{0 n}} & -\frac{\partial \varphi_{n-1}}{\partial \lambda} \\
-\frac{\partial \varphi_{n}}{\partial x_{01}} & \cdots & \cdots & 1-\frac{\partial \varphi_{n}}{\partial x_{0 n}} & -\frac{\partial \varphi_{n}}{\partial \lambda} \\
\frac{\partial \chi}{\partial x_{01}} & \cdots & \cdots & \frac{\partial \chi}{\partial x_{0 n}} & \frac{\partial \chi}{\partial \lambda}
\end{array}\right) .
\end{aligned}
$$

Readers already know how to obtain derivatives like $\partial \varphi / \partial x_{0}$. These are of course, computed as numerical solutions to the variational equation Eq. (36). The question now is, how do we obtain the rest of the derivatives like $\partial \varphi_{i} / \partial \lambda$ and the derivatives of the characteristic equation $\chi\left(x_{0}, \lambda, \mu^{*}\right)$ with respect to the initial value $x_{0}$ and the parameter $\lambda$ ?

First, let us consider the derivative with respect to a parameter, $\partial \varphi_{i} / \partial \lambda$. For Eq. (32), we assume a solution including $\lambda$ as a parameter:

$$
x(t)=\varphi\left(t, \lambda ; t_{0}, x_{0}\right)
$$

Differentiating both sides of this equation with respect to $\lambda$ yields

$$
\frac{\partial x}{\partial \lambda}=\frac{\partial \varphi}{\partial \lambda}
$$

Substituting this into Eq. (32), we get a linear differential equation:

$$
\frac{d}{d t}\left(\frac{\partial \varphi}{\partial \lambda}\right)=\frac{\partial f}{\partial x} \frac{\partial \varphi}{\partial \lambda}+\frac{\partial f}{\partial \lambda}
$$

This is the variational equation with respect to the parameter, $\lambda$. In the same way as computation of the variational equation (Eq. (36)), we can obtain the solution by using a suitable numerical integration method. 
Next let us consider the derivatives of the characteristic equation. The characteristic equation $\chi\left(x_{0}, \lambda, \mu^{*}\right)$ is the determinant of the following $n \times n$ matrix:

$$
P\left(x_{0}\right):=\frac{\partial S_{\lambda}}{\partial x_{0}}-\mu^{*} I=\left(\begin{array}{cccc}
\frac{\partial \varphi_{1}}{\partial x_{01}}-\mu^{*} & \frac{\partial \varphi_{1}}{\partial x_{02}} & \cdots & \frac{\partial \varphi_{1}}{\partial x_{0 n}} \\
\frac{\partial \varphi_{2}}{\partial x_{01}} & \ddots & & \vdots \\
\vdots & & \ddots & \frac{\partial \varphi_{n-1}}{\partial x_{0 n}} \\
\frac{\partial \varphi_{n}}{\partial x_{01}} & \cdots & \cdots & \frac{\partial \varphi_{n}}{\partial x_{0 n}}-\mu^{*}
\end{array}\right),
$$

i.e., $\chi\left(x_{0}, \lambda, \mu^{*}\right):=\operatorname{det}(P)=0$. In Eq. (42), the derivatives of the characteristic equation with respect to the initial value $x_{0}$ can be obtained by using the following formula [27]

$$
\frac{\partial \chi\left(x_{0}, \lambda, \mu^{*}\right)}{\partial x_{0}}=\sum_{i=1}^{n} \operatorname{det}\left(P_{i}\right),
$$

where $P_{i}$ are matrices that are differentiated from each element of the $i$ th column of $P$ with regard to $x_{0}$. As an example, if the function of Eq. (32) has a state vector $x=\left(x_{1}, x_{2}, x_{3}\right)^{\top} \in R^{3}$, the derivative of the characteristic equation with respect to the element $x_{01}$ in the initial value $x_{0}=\left(x_{01}, x_{02}, x_{03}\right)^{\top}$, is as follows:

$$
\begin{aligned}
\frac{\partial \chi\left(x_{0}, \lambda, \mu^{*}\right)}{\partial x_{01}} & =\sum_{i=1}^{3} \operatorname{det}\left(P_{i}\right)=\left|\begin{array}{lll}
\frac{\partial}{\partial x_{01}}\left(\frac{\partial \varphi_{1}}{\partial x_{01}}-\mu^{*}\right) & \frac{\partial}{\partial x_{01}} \frac{\partial \varphi_{1}}{\partial x_{02}} & \frac{\partial}{\partial x_{01}} \frac{\partial \varphi_{1}}{\partial x_{03}} \\
\frac{\partial \varphi_{01}}{\partial \varphi_{3}} & \frac{\partial \varphi_{2}}{\partial x_{02}}-\mu^{*} & \frac{\partial \varphi_{2}}{\partial x_{03}} \\
\frac{\partial \varphi_{3}}{\partial x_{02}} & \frac{\partial \varphi_{3}}{\partial x_{03}}-\mu^{*}
\end{array}\right| \\
& +\left|\begin{array}{lll}
\frac{\partial \varphi_{1}}{\partial x_{01}}-\mu^{*} & \frac{\partial \varphi_{1}}{\partial x_{02}} & \frac{\partial \varphi_{1}}{\partial x_{03}} \\
\frac{\partial}{\partial x_{01}} \frac{\partial \varphi_{2}}{\partial x_{01}} & \frac{\partial}{\partial x_{01}}\left(\frac{\partial \varphi_{2}}{\partial x_{02}}-\mu^{*}\right) & \frac{\partial}{\partial x_{01}} \frac{\partial \varphi_{2}}{\partial x_{03}} \\
\frac{\partial \varphi_{3}}{\partial x_{01}} & \frac{\partial \varphi_{3}}{\partial x_{02}} & \frac{\partial \varphi_{3}}{\partial x_{03}}-\mu^{*}
\end{array}\right| \\
& +\left|\begin{array}{llll}
\frac{\partial \varphi_{1}}{\partial x_{01}}-\mu^{*} & \frac{\partial \varphi_{1}}{\partial x_{02}} & \frac{\partial \varphi_{1}}{\partial x_{03}} & \\
\frac{\partial \varphi_{2}}{\partial x_{01}} & \frac{\partial \varphi_{2}}{\partial x_{02}}-\mu^{*} & \frac{\partial \varphi_{2}}{\partial x_{03}} & \\
\frac{\partial}{\partial x_{01}} \frac{\partial \varphi_{3}}{\partial x_{01}} & \frac{\partial}{\partial x_{01}} \frac{\partial \varphi_{3}}{\partial x_{02}} & \frac{\partial}{\partial x_{01}}\left(\frac{\partial \varphi_{3}}{\partial x_{03}}-\mu^{*}\right)
\end{array}\right|
\end{aligned}
$$

The derivatives of the characteristic equation related to the parameter $\lambda$ are the same as those in Eq. (47). Consequently, to calculate each element of the Jacobian matrix of Eq. (42), it is necessary to obtain the second derivatives of the Poincaré map $S_{\lambda}$ for $x_{0}$ :

$$
\frac{\partial}{\partial x_{0 j}}\left(\frac{\partial S_{\lambda}}{\partial x_{0}}\right)=\frac{\partial}{\partial x_{0 j}}\left(\frac{\partial \varphi}{\partial x_{0}}\right),
$$

for $j=1,2, \ldots, n$, where $x_{0 j}$ denotes an element in the initial value $x_{0}$. Let us differentiate Eq. (36) with respect to $x_{0}$ one more time:

$$
\frac{d}{d t}\left\{\frac{\partial}{\partial x_{0 j}}\left(\frac{\partial \varphi}{\partial x_{0}}\right)\right\}=\frac{\partial f}{\partial x_{0}} \frac{\partial}{\partial x_{0 j}}\left(\frac{\partial \varphi}{\partial x_{0}}\right)+\frac{\partial}{\partial x_{0 j}}\left(\frac{\partial f}{\partial x_{0}}\right)\left(\frac{\partial \varphi}{\partial x_{0}}\right)^{2},
$$

for $j=1,2, \ldots, n$. This equation is also linear. The numerical solution of Eq. (36) can be substituted for $\partial \varphi / \partial x_{0}$. The underlined part is a tensor. Even though its expanded form seems very complicated, it can be computed by numerical integration. We call this equation the second variational equation. The Appendix describes its expanded forms in detail.

All of the elements in the Jacobian matrix in Eq. (42) are now ready to be computed. 


\subsection{Locating fixed points, bifurcations}

Our scheme proceeds as follows:

1. First, use numerical simulations to find the location of a stable fixed point. If it is the initially chosen point is already stable, the fixed point locating program does not have to be run. By freely changing parameter values, you can browse several phenomena before doing the computation. If you encounter a bifurcation, save the parameter values and the location of the Poincaré mapping points.

2. Use Newton's method, Eq. (28), to compute the fixed point accurately. Next, try Newton's method again with this result and a slightly changed parameter value. The new parameter value will probably lead to a new location. This method is called continuation. Just before a period-doubling bifurcation, near the parameter values memorized above, one of the multipliers is greater than or approximately equal to -1 , i.e., $\mu_{i} \gtrsim-1$ and this negative value grows as the parameter changes. For a tangent bifurcation, the continuation fails near the bifurcation parameter value since the Jacobian matrix in Eq. (29) is singular and $\mu_{i}$ is just slightly less than unity. For a Neimark-Sacker bifurcation, the absolute value of the complex conjugate multipliers exceed the unit circle and are approximately unity, i.e., $\left|\mu_{i}\right| \approx 1$.

3. Execute Newton's method, Eq. (39), using the state variables and the parameter values just before/after the bifurcations identified above. If the iterations converge, the final value is an accurate bifurcation parameter. To obtain a two-parameter bifurcation diagram, another parameter is varied slightly, and Newton's method is executed again using the last obtained fixed point and parameter values. This procedure is also called continuation.

\subsection{Example - Duffing Rayleigh equation}

Here, we shall numerically analyze the bifurcations observed in the Duffing Rayleigh equation, a typical example of a non-autonomous system. The Duffing Rayleigh equation describes the dynamics of a spring containing nonlinear characteristics:

$$
\begin{aligned}
& \frac{d x}{d t}=y \\
& \frac{d y}{d t}=\epsilon\left(1-y^{2}\right) y-x^{3}+B \cos \nu t .
\end{aligned}
$$

For $B=0$, the system is a van der Pol oscillator. There is an unstable origin equilibrium point and a stable limit cycle with $\epsilon>0$. Thus, for a certain value of $B$, two frequency components compete and synchronize with each other. The system may contain a rich variety of bifurcation sets in the parameter space because of the frequency difference between the two frequency components; there are frequency entrainment regions called "Arnold tongues" surrounded by tangent bifurcation sets. A quasi-periodic solution is obtained via the Neimark-Sacker bifurcation, whereas a periodic solution in the Arnold tongue may encounter period-doubling bifurcation cascades.

Figure 2 shows a bifurcation diagram of the Duffing-Rayleigh equation in the $\nu$ - $B$ plane with $\epsilon=0.2$. Let us assume that $\epsilon$ and $\nu$ are fixed parameters. By applying the formulas (A-4) and (A-14) to Eq. (51), we can obtain the first and second variational equations (see Table II). The variation of $B$ is given in Table II. $\nu$ should be an incremental parameter; however, it is hard to track the bifurcation sets with monotonic increments in a two-dimensional parameter space since the bifurcation sets generally form a 'curve'. In this case, the variational parameter and incremental parameter can be adaptively switched. Note that one should rescale the time as $\tau=\nu t$ to solve Eq. (39) with the variational parameter $\nu$.

$G, I$ and NS in Fig. 2 show tangent, period-doubling, and Neimark-Sacker bifurcations, respectively. Their superscripts indicates the period of the fixed/periodic point, and subscripts show the nominal numbers.

There is a period-1 entrainment region, and it is divided up into sections by Neimark-Sacker and tangent bifurcation sets. Moreover, there are various frequency entrainment (period locking) regions 
Table II. The variational equations of the Duffing equation.

\begin{tabular}{l|c|l|c}
\hline \multicolumn{1}{c|}{ variables } & $\begin{array}{c}\text { computer } \\
\text { variables }\end{array}$ & \multicolumn{1}{|c}{ differential eq. } & initial values \\
\hline \hline$x=\varphi_{1}\left(t, x_{0}, y_{0}, \epsilon\right)$ & $x_{1}$ & $\dot{x}_{1}=x_{2}$ & $x_{0}$ \\
$y=\varphi_{2}\left(t, x_{0}, y_{0}, \epsilon\right)$ & $x_{2}$ & $\dot{x}_{2}=\epsilon\left(1-x_{2}^{2}\right) x_{2}-x_{1}^{3}+B \cos \nu t$ & $y_{0}$ \\
\hline$\partial \varphi_{1} / \partial x_{0}$ & $x_{3}$ & $\dot{x}_{3}=x_{4}$ & 1 \\
$\partial \varphi_{2} / \partial x_{0}$ & $x_{4}$ & $\dot{x}_{4}=P x_{3}+Q x_{4}$ & 0 \\
$\partial \varphi_{1} / \partial y_{0}$ & $x_{5}$ & $\dot{x}_{5}=x_{6}$ & 1 \\
$\partial \varphi_{2} / \partial y_{0}$ & $x_{6}$ & $\dot{x}_{6}=P x_{5}+Q x_{6}$ & 0 \\
\hline$\varphi_{1} / \partial B$ & $x_{7}$ & $\dot{x}_{7}=x_{8}$ & 0 \\
$\partial \varphi_{2} / \partial B$ & $x_{8}$ & $\dot{x}_{8}=P x_{7}+Q x_{8}+\cos \nu t$ & 0 \\
\hline$\partial^{2} \varphi_{1} / \partial x_{0}^{2}$ & $x_{9}$ & $\dot{x}_{9}=x_{10}$ & 0 \\
$\partial^{2} \varphi_{2} / \partial x_{0}^{2}$ & $x_{10}$ & $\dot{x}_{10}=P x_{9}+Q x_{10}+R x_{3}^{2}+S x_{4}^{2}$ & 0 \\
$\partial^{2} \varphi_{1} / \partial x_{0} \partial y_{0}$ & $x_{11}$ & $\dot{x}_{11}=x_{12}$ & 0 \\
$\partial^{2} \varphi_{2} / \partial x_{0} \partial y_{0}$ & $x_{12}$ & $\dot{x}_{12}=P x_{11}+Q x_{12}+R x_{3} x_{5}+S x_{4} x_{6}$ & 0 \\
$\partial^{2} \varphi_{1} / \partial y_{0}^{2}$ & $x_{13}$ & $\dot{x}_{13}=x_{14}$ & 0 \\
$\partial^{2} \varphi_{2} / \partial y_{0}^{2}$ & $x_{14}$ & $\dot{x}_{14}=P x_{13}+Q x_{14}+R x_{5}^{2}+S x_{6}^{2}$ & 0 \\
\hline$\partial^{2} \varphi_{1} / \partial x_{0} \partial B$ & $x_{15}$ & $\dot{x}_{15}=x_{16}$ & 0 \\
$\partial^{2} \varphi_{2} / \partial x_{0} \partial B$ & $x_{16}$ & $\dot{x}_{16}=P x_{15}+Q x_{16}+R x_{3} x_{7}+S x_{4} x_{8}$ & 0 \\
$\partial^{2} \varphi_{1} / \partial y_{0} \partial B$ & $x_{17}$ & $\dot{x}_{17}=x_{18}$ & \\
$\partial^{2} \varphi_{2} / \partial y_{0} \partial B$ & $x_{18}$ & $\dot{x}_{18}=P x_{17}+Q x_{18}+R x_{5} x_{7}+S x_{6} x_{8}$ & \\
\hline$P=-3 x_{1}^{2}, Q=\epsilon\left(1-3 x_{2}^{2}\right), R=-6 x_{1}, S=-6 \epsilon x_{2}$. & \\
\hline
\end{tabular}

surrounded by tangent bifurcations, among them, quasi-periodic motions called beat motions and 2-torus solutions.

Figure 3 is an enlargement of Fig. 2. Around the period-2 entrainment region, there are other odd-number period regions and they overlap each other; i.e., periodic solutions with the same parameter values coexist and their appearance depends on the initial value. Period-doubling cascades are frequently found for such periodic solutions, e.g., $I_{1}^{2}$, and $I_{1}^{4}$; one can also find $I_{1}^{2^{k}}, k=3,4,5, \ldots$, near $I_{1}^{4}$.

\subsection{Extensions of bifurcation analysis}

The above bifurcation analysis is the results of many improvements. In this subsection, we will present several extensions to Kawakami's shooting method [5].

\subsubsection{Neimark-Sacker bifurcation}

The Neimark-Sacker bifurcation occurs when the complex conjugate multipliers $\mu_{i, i^{\prime}}$ move out of the unit circle transversely as a parameter changes. As shown in subsection 3.2, the bifurcation parameter is obtained to solve for the conditions of the fixed point and the characteristic equation simultaneously (see Eq. (39)). However, the characteristic equation, $\operatorname{det}\left(D S_{\lambda}\left(x_{0}^{*}\right)-\mu^{*} I\right)=0$, is not a suitable condition for the calculating a Neimark-Sacker bifurcation parameter [13], since the multiplier is a complex number with argument $\theta$. Therefore, we can redefine the conditions describing Neimark-Sacker bifurcation as:

$$
F_{N S}:=\left[\begin{array}{c}
x_{0}-S_{\lambda}\left(x_{0}\right) \\
\Re\left[\operatorname{det}\left(D S_{\lambda}\left(x_{0}^{*}\right)-e^{j \theta} I\right)\right] \\
\Im\left[\operatorname{det}\left(D S_{\lambda}\left(x_{0}^{*}\right)-e^{j \theta} I\right)\right]
\end{array}\right]=0,
$$

Accordingly, we can simultaneously solve Eq. (52) for $u=\left(x_{0}, \lambda, \theta\right)$ with Newton's method. The Jacobian matrix utilized in Newton's method is as follows: 


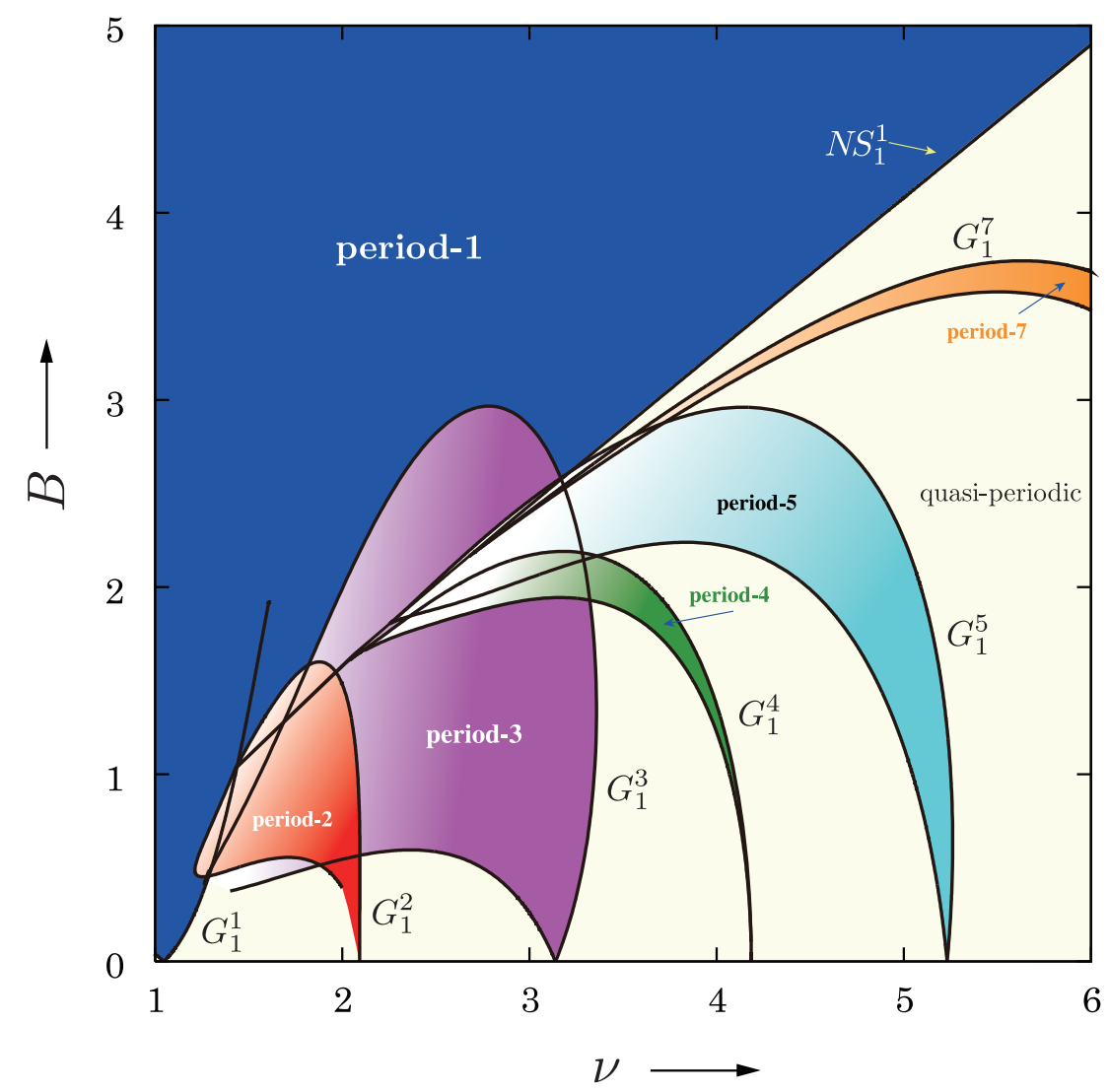

Fig. 2. Bifurcation sets of periodic solutions to the Duffing Rayleigh equation in the $\nu$ - $B$ plane.

$$
D F_{N S}\left(u^{(k)}\right)=\left(\begin{array}{cccccc}
1-\frac{\partial \varphi_{1}}{\partial x_{01}} & -\frac{\partial \varphi_{1}}{\partial x_{02}} & \cdots & -\frac{\partial \varphi_{1}}{\partial x_{0 n}} & -\frac{\partial \varphi_{1}}{\partial \lambda} & -\frac{\partial \varphi_{1}}{\partial \theta} \\
-\frac{\partial \varphi_{2}}{\partial x_{01}} & \ddots & \vdots & \vdots & \vdots \\
\vdots & & \ddots & -\frac{\partial \varphi_{n-1}}{\partial x_{0 n}} & -\frac{\partial \varphi_{n-1}}{\partial \lambda} & -\frac{\partial \varphi_{n-1}}{\partial \theta} \\
-\frac{\partial \varphi_{n}}{\partial x_{01}} & \ldots & \ldots & 1-\frac{\partial \varphi_{n}}{\partial x_{0 n}} & -\frac{\partial \varphi_{n}}{\partial \lambda} & -\frac{\partial \varphi_{n}}{\partial \theta} \\
\Re\left(\frac{\partial \chi}{\partial x_{01}}\right) & \ldots & \ldots & \Re\left(\frac{\partial \chi}{\partial x_{0 n}}\right) & \Re\left(\frac{\partial \chi}{\partial \lambda}\right) & \Re\left(\frac{\partial \chi}{\partial \theta}\right) \\
\Im\left(\frac{\partial \chi}{\partial x_{01}}\right) & \ldots & \ldots & \Im\left(\frac{\partial \chi}{\partial x_{0 n}}\right) & \Im\left(\frac{\partial \chi}{\partial \lambda}\right) & \Im\left(\frac{\partial \chi}{\partial \theta}\right)
\end{array}\right)
$$

We should note that $\partial \varphi / \partial \theta$ can be regarded as 0 since the solution $\varphi$ is not related to the argument of the complex multipliers. This means that the complex argument is neither an independent variable nor a parameter for Newton's method. However, most computer languages cannot manipulate complex variables directly. Therefore, Ueta et al., [28, 29] have proposed an extension in which the derivatives of the real and imaginary parts of the characteristic equation are calculated with simple matrix operations by utilizing the multi-linearity of the determinant and the solutions of the variational equations [28,29]. A script language, e.g., Mathematica, MATLAB, etc., may be quite useful because it can directly deal with complex variables and matrix operations.

\subsubsection{Discontinuous periodic forcing}

Here, we will briefly introduce an extended method for analyzing bifurcations in a discontinuous dynamical system $[14,15,30-32]$. In particular, we will focus on a non-autonomous system driven by a discontinuous periodic force, e.g., an impulsive pulse train [30], square-wave train [31], or trapezoidal wave train [32]. Such systems have been modeled as piecewise smooth differential equations switched by a periodic interrupt. In this case, the system can be defined as a composite dynamical system. 


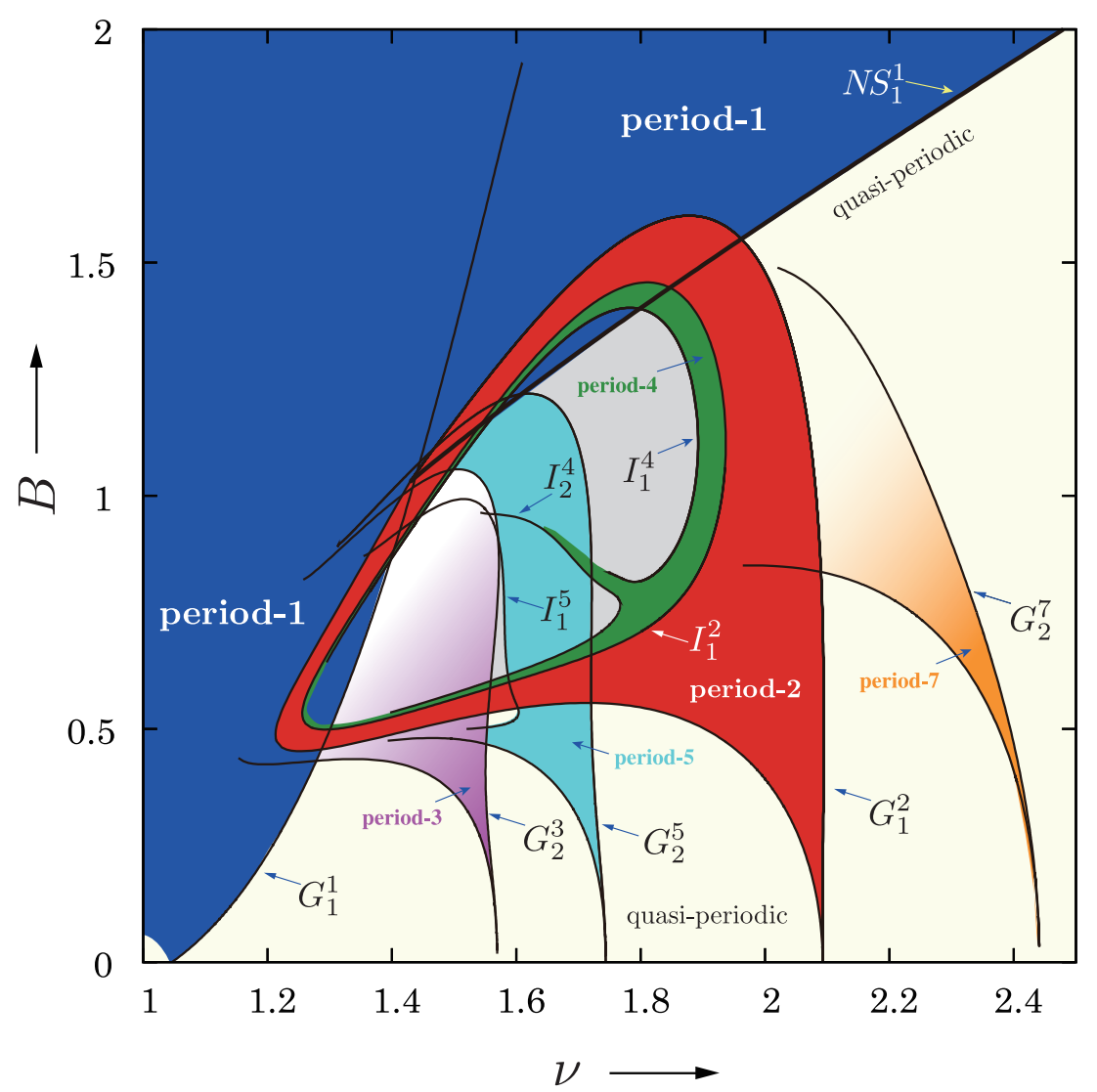

Fig. 3. Enlargement of Fig. 2.

Even though the trajectory in the composite system has a discontinuous nature at the moment of switching, by avoiding differentiation of discontinuous points that appear in the solution, a Poincaré map can be constructed from successive submaps. Therefore, we can directly calculate the bifurcation values from the original equations without having to use a special coordinate transformation.

Now, let us assume that a parameter periodically switches from a low value during $t_{0} \leq t<t_{0}+\tau_{a}$ to a high value during $t_{0}+\tau_{a} \leq t<t_{0}+\tau_{p}$, and vice versa (see Fig. 4). The dynamics of Eq. (2) during the low (resp., high) value period are the same as the autonomous system of Eq. (1) except for the parameter $\lambda_{s}$, with the constant parameter $\lambda_{s}^{L}$ (resp., $\lambda_{s}^{H}$ ). Thus, Eq. (2) can be defined as a composite dynamical system so that the two corresponding autonomous systems can be periodically switched over time.

Let us rewrite the system of Eq. (2) during a period satisfying $t-t_{0}\left(\bmod \tau_{p}\right) \in\left[0, \tau_{p}\right)$ as

$$
\frac{d x}{d t}=f(t, x, \lambda)=\left\{\begin{array}{cc}
f_{L}\left(x, \lambda_{0}, \lambda_{s}^{L}\right), & t_{0} \leq t<t_{0}+\tau_{a} \\
f_{H}\left(x, \lambda_{0}, \lambda_{s}^{H}\right), & t_{0}+\tau_{a} \leq t<t_{0}+\tau_{p},
\end{array}\right.
$$

where $\lambda_{0} \in R^{\ell-1}$ denotes common parameters for $f$, and $\lambda_{s}^{L}, \lambda_{s}^{H} \in R$ are the parameters specifying $f_{L}$ and $f_{H}$, respectively. Suppose that the whole solution of Eq. (54) is described as a mixed solution of the first and second equations of Eq. (54). Then the solution is represented by

$$
x(t)=\varphi\left(t, \lambda ; t_{0}, x_{0}\right)=\varphi\left(t, \lambda_{0}, \lambda_{s}^{L}, \lambda_{s}^{H} ; t_{0}, x_{0}\right) .
$$

As shown in Fig. 4, the trajectory of the solution has a discontinuous point. Let $\varphi_{L}$ and $\varphi_{H}$ be solutions to the first and second equations of Eq. (54), respectively:

$$
x(t)=\varphi_{L}\left(t, \lambda_{0}, \lambda_{s}^{L} ; t_{0}, x_{0}\right), \quad t_{0} \leq t<t_{0}+\tau_{a},
$$

and

$$
x(t)=\varphi_{H}\left(t, \lambda_{0}, \lambda_{s}^{H} ; t_{0}+\tau_{a}, \varphi_{L}\left(t_{0}+\tau_{a}, \lambda_{0}, \lambda_{s}^{L} ; t_{0}, x_{0}\right)\right), \quad t_{0}+\tau_{a} \leq t<t_{0}+\tau_{p} .
$$




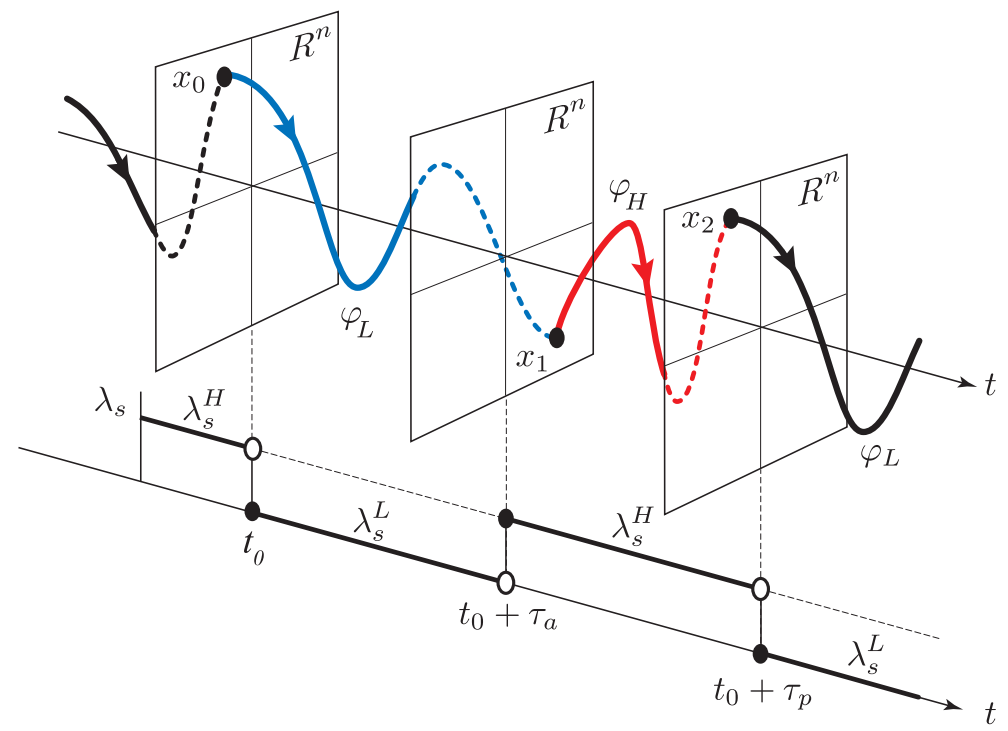

Fig. 4. Schematic diagram of a discontinuous trajectory.

To deal with the impossibility of the derivative at $t=t_{0}$ and $t=t_{0}+\tau_{a}$, we use the Poincaré map,

$$
\begin{aligned}
S_{d}: & R^{n} \\
x_{0} & \mapsto S_{d}\left(x_{0}\right)=\varphi\left(t_{0}+\tau_{p}, \lambda_{0}, \lambda_{s}^{L}, \lambda_{s}^{H} ; t_{0}, x_{0}\right)
\end{aligned}
$$

as a composite map $S_{d}=S_{d 2} \circ S_{d 1}$, where $S_{d 1}$ and $S_{d 2}$ are the following submaps:

$$
\begin{aligned}
S_{d 1}: \quad R^{n} & \rightarrow R^{n} \\
x_{0} & \mapsto x_{1}=\varphi_{L}\left(t_{0}+\tau_{a}, \lambda_{0}, \lambda_{s}^{L} ; t_{0}, x_{0}\right) \\
S_{d 2}: \quad R^{n} & \rightarrow R^{n} \\
x_{1} & \mapsto x_{2}=\varphi_{H}\left(t_{0}+\tau_{p}, \lambda_{0}, \lambda_{s}^{H} ; t_{0}+\tau_{a}, x_{1}\right) .
\end{aligned}
$$

The first derivative of the Poincaré map $S_{d}$ of Eq. (58) with respect to the initial value $x_{0}$ is composed of the derivatives of the submaps, i.e.,

$$
\begin{aligned}
\frac{\partial S_{d}\left(x_{0}\right)}{\partial x_{0}} & =\frac{\partial S_{d 2}\left(x_{1}\right)}{\partial x_{1}} \frac{\partial S_{d 1}\left(x_{1}\right)}{\partial x_{0}} \\
& =\frac{\partial \varphi_{H}}{\partial x_{1}}\left(t_{0}+\tau_{p}, \lambda_{0}, \lambda_{s}^{H} ; t_{0}+\tau_{a}, x_{1}\right) \times \frac{\partial \varphi_{L}}{\partial x_{0}}\left(t_{0}+\tau_{a}, \lambda_{0}, \lambda_{s}^{L} ; t_{0}, x_{0}\right) .
\end{aligned}
$$

The right side of the above equation (60) is obtained by solving the following first-order variational equations:

$$
\begin{aligned}
& \frac{d}{d t}\left(\frac{\partial \varphi_{L}}{\partial x_{0}}\right)=\frac{\partial f_{L}}{\partial x}\left(\frac{\partial \varphi_{L}}{\partial x_{0}}\right) \quad \text { with }\left.\frac{\partial \varphi_{L}}{\partial x_{0}}\right|_{t=t_{0}}=I, \\
& \frac{d}{d t}\left(\frac{\partial \varphi_{H}}{\partial x_{1}}\right)=\frac{\partial f_{H}}{\partial x}\left(\frac{\partial \varphi_{H}}{\partial x_{1}}\right) \quad \text { with }\left.\quad \frac{\partial \varphi_{H}}{\partial x_{1}}\right|_{t=t_{0}+\tau_{a}}=I,
\end{aligned}
$$

and putting $t=t_{0}+\tau_{a}$ and $t_{0}+\tau_{p}$ in the solutions of Eqs. (61) and (62), respectively.

As shown in the previous subsection, we must also compute the first and second derivatives with respect to a bifurcation parameter and the initial value $x_{0}$. If we choose a common parameter $\lambda_{c} \in \lambda_{0}$ except for the specific parameter $\lambda_{s}^{L}, \lambda_{s}^{H}$ in Eq. (54), the derivatives are as follows:

$$
\frac{\partial S_{d}}{\partial \lambda_{c}}=\frac{\partial \varphi_{H}}{\partial \lambda_{c}}\left(t_{0}+\tau_{p}, \lambda_{0}, \lambda_{s}^{H} ; t_{0}+\tau_{a}, x_{1}\right)=\frac{\partial \varphi_{H}}{\partial \lambda_{c}}+\frac{\partial \varphi_{H}}{\partial x_{1}} \frac{\partial \varphi_{L}}{\partial \lambda_{c}}
$$

On the one hand, the following formula are obtained by differentiating with respect to the specific parameters $\lambda_{s}^{L}$ and $\lambda_{s}^{H}$ : 


$$
\begin{aligned}
\frac{\partial S_{d}}{\partial \lambda_{s}^{L}} & =\frac{\partial \varphi_{H}}{\partial \lambda_{s}^{L}}+\frac{\partial \varphi_{H}}{\partial x_{1}} \frac{\partial \varphi_{L}}{\partial \lambda_{s}^{L}}=\frac{\partial \varphi_{H}}{\partial x_{1}} \frac{\partial \varphi_{L}}{\partial \lambda_{s}^{L}} \\
\frac{\partial S_{d}}{\partial \lambda_{s}^{H}} & =\frac{\partial \varphi_{H}}{\partial \lambda_{s}^{H}}+\frac{\partial \varphi_{H}}{\partial x_{1}} \frac{\partial \varphi_{L}}{\partial \lambda_{s}^{H}}=\frac{\partial \varphi_{H}}{\partial \lambda_{s}^{H}}
\end{aligned}
$$

On the other hand, we get the second derivatives of $S_{d}$ with respect to $x_{0}$, that is $\partial / \partial x_{0}\left(\partial S_{d} / \partial x_{0}\right)$, and with respect to the bifurcation parameter $\lambda_{1}$, i.e., $\partial / \partial \lambda_{1}\left(\partial S_{d} / \partial x_{0}\right)$, depending on the parameter chosen, for instance, $\lambda_{c}$ or $\lambda_{s}^{L}$ or $\lambda_{s}^{H}$. The calculations of the second derivatives of $S_{d}$ with respect to the initial value, and bifurcation parameter are the same as Eqs. (49) and (50).

The above enables us to obtain bifurcation values directly from the original equations without making a special coordinate transformation. Hence, we can easily trace out various bifurcation sets in an appropriate parameter plane.

\section{Conclusions}

We briefly outlined bifurcation theory and described in detail a method for performing the numerical computations of bifurcation analysis in a non-autonomous system with a periodic force. The variational equations play critical roles in this shooting method for calculating bifurcation sets. The variational equation can be obtained by using various numerical differentiation methods but, such methods are greatly affected by numerical errors. If the values of the first variational equations include any numerical error, the multiplier of the characteristic equation will not be accurate. In such circumstances, the first variational equation, at least, should be described explicitly. Our method can obtain a precise bifurcation set without incurring any of the worries involved in the various numerical differentiation methods. In fact, we have developed an integrated analysis environment named "BunKi" under the support of Aihara Complexity Modeling Project, ERATO, JST [33]. The system implements the simple shooting method described in this article on a MATLAB platform.

Researchers and graduate students of nonlinear science and bifurcation theory should be able to understand the principles of the algorithms they use for solving bifurcation problems, or else they will not be able to understand, let alone explain to others, the results of their analysis tools. Moreover, such a lack of understanding makes it virtually impossible to extend an algorithm when needed. We hope this article will help readers understand the nature of bifurcation analysis in dynamical systems.

\section{Appendix}

\section{A. Variational equations for 2-dimensional non-autonomous systems}

Consider a two-dimensional differential equation including a parameter:

$$
\begin{aligned}
& \frac{d x}{d t}=f_{1}(t, x, y, \lambda) \\
& \frac{d y}{d t}=f_{2}(t, x, y, \lambda)
\end{aligned}
$$

The solution of Eq. (A-1) passing through $\left(x_{0}, y_{0}\right)$ at $t=0$ can be described as follows:

$$
\begin{aligned}
& x(t)=\varphi_{1}\left(t, x_{0}, y_{0}, \lambda\right) \\
& y(t)=\varphi_{2}\left(t, x_{0}, y_{0}, \lambda\right) .
\end{aligned}
$$

That is,

$$
\begin{aligned}
& \frac{d \varphi_{1}}{d t}=f_{1}\left(t, \varphi_{1}, \varphi_{2}, \lambda\right) \\
& \frac{d \varphi_{2}}{d t}=f_{2}\left(t, \varphi_{1}, \varphi_{2}, \lambda\right) \\
& \varphi_{1}\left(0, x_{0}, y_{0}, \lambda\right)=x_{0} \\
& \varphi_{2}\left(0, x_{0}, y_{0}, \lambda\right)=y_{0} .
\end{aligned}
$$




\section{A.1 The first variational equation}

$$
\begin{array}{r}
\frac{d}{d t}\left(\begin{array}{c}
\frac{\partial \varphi_{1}}{\partial x_{0}} \\
\frac{\partial \varphi_{2}}{\partial x_{0}}
\end{array}\right)=\left(\begin{array}{ll}
\frac{\partial f_{1}}{\partial x} & \frac{\partial f_{1}}{\partial y} \\
\frac{\partial f_{2}}{\partial x} & \frac{\partial f_{2}}{\partial y}
\end{array}\right)\left(\begin{array}{c}
\frac{\partial \varphi_{1}}{\partial x_{0}} \\
\frac{\partial \varphi_{2}}{\partial x_{0}}
\end{array}\right),\left.\quad\left(\begin{array}{c}
\frac{\partial \varphi_{1}}{\partial x_{0}} \\
\frac{\partial \varphi_{2}}{\partial x_{0}}
\end{array}\right)\right|_{t=0}=\left(\begin{array}{c}
1 \\
0
\end{array}\right) \\
\frac{d}{d t}\left(\begin{array}{c}
\frac{\partial \varphi_{1}}{\partial y_{0}} \\
\frac{\partial \varphi_{2}}{\partial y_{0}}
\end{array}\right)=\left(\begin{array}{cc}
\frac{\partial f_{1}}{\partial x} & \frac{\partial f_{1}}{\partial y} \\
\frac{\partial f_{2}}{\partial x} & \frac{\partial f_{2}}{\partial y}
\end{array}\right)\left(\begin{array}{c}
\frac{\partial \varphi_{1}}{\partial y_{0}} \\
\frac{\partial \varphi_{2}}{\partial y_{0}}
\end{array}\right),\left.\quad\left(\begin{array}{c}
\frac{\partial \varphi_{1}}{\partial y_{0}} \\
\frac{\partial \varphi_{2}}{\partial y_{0}}
\end{array}\right)\right|_{t=0}=\left(\begin{array}{c}
0 \\
1
\end{array}\right) \\
\frac{d}{d t}\left(\begin{array}{c}
\frac{\partial \varphi_{1}}{\partial \lambda} \\
\frac{\partial \varphi_{2}}{\partial \lambda}
\end{array}\right)=\left(\begin{array}{ll}
\frac{\partial f_{1}}{\partial x} & \frac{\partial f_{1}}{\partial y} \\
\frac{\partial f_{2}}{\partial x} & \frac{\partial f_{2}}{\partial y}
\end{array}\right)\left(\begin{array}{c}
\frac{\partial \varphi_{1}}{\partial \lambda} \\
\frac{\partial \varphi_{2}}{\partial \lambda}
\end{array}\right)+\left(\begin{array}{c}
\frac{\partial f_{1}}{\partial \lambda} \\
\frac{\partial f_{2}}{\partial \lambda}
\end{array}\right),\left.\quad\left(\begin{array}{c}
\frac{\partial \varphi_{1}}{\partial \lambda} \\
\frac{\partial \varphi_{2}}{\partial \lambda}
\end{array}\right)\right|_{t=0}=\left(\begin{array}{c}
0 \\
0
\end{array}\right)
\end{array}
$$

\section{A.2 The second variational equation}

$$
\begin{aligned}
& \frac{d}{d t}\left(\begin{array}{c}
\frac{\partial^{2} \varphi_{1}}{\partial x_{0}^{2}} \\
\frac{\partial^{2} \varphi_{2}}{\partial x_{0}^{2}}
\end{array}\right)=D f\left(\begin{array}{c}
\frac{\partial^{2} \varphi_{1}}{\partial x_{0}^{2}} \\
\frac{\partial^{2} \varphi_{2}}{\partial x_{0}^{2}}
\end{array}\right)+\left(\frac{\partial}{\partial x_{0}} D f\right)\left(\begin{array}{c}
\frac{\partial \varphi_{1}}{\partial x_{0}} \\
\frac{\partial \varphi_{2}}{\partial x_{0}}
\end{array}\right) \\
& \frac{d}{d t}\left(\begin{array}{c}
\frac{\partial^{2} \varphi_{1}}{\partial x_{0} \partial y_{0}} \\
\frac{\partial^{2} \varphi_{2}}{\partial x_{0} \partial y_{0}}
\end{array}\right)=D f\left(\begin{array}{c}
\frac{\partial^{2} \varphi_{1}}{\partial x_{0} \partial y_{0}} \\
\frac{\partial^{2} \varphi_{2}}{\partial x_{0} \partial y_{0}}
\end{array}\right)+\left(\frac{\partial}{\partial x_{0}} D f\right)\left(\begin{array}{c}
\frac{\partial \varphi_{1}}{\partial y_{0}} \\
\frac{\partial \varphi_{2}}{\partial y_{0}}
\end{array}\right) \\
& \frac{d}{d t}\left(\begin{array}{c}
\frac{\partial^{2} \varphi_{1}}{\partial y_{0}^{2}} \\
\frac{\partial^{2} \varphi_{2}}{\partial y_{0}^{2}}
\end{array}\right)=D f\left(\begin{array}{c}
\frac{\partial^{2} \varphi_{1}}{\partial y_{0}^{2}} \\
\frac{\partial^{2} \varphi_{2}}{\partial y_{0}^{2}}
\end{array}\right)+\left(\frac{\partial}{\partial y_{0}} D f\right)\left(\begin{array}{c}
\frac{\partial \varphi_{1}}{\partial y_{0}} \\
\frac{\partial \varphi_{2}}{\partial y_{0}}
\end{array}\right) \\
& \frac{d}{d t}\left(\begin{array}{c}
\frac{\partial^{2} \varphi_{1}}{\partial x_{0} \partial \lambda} \\
\frac{\partial^{2} \varphi_{2}}{\partial x_{0} \partial \lambda}
\end{array}\right)=D f\left(\begin{array}{c}
\frac{\partial^{2} \varphi_{1}}{\partial x_{0} \partial \lambda} \\
\frac{\partial^{2} \varphi_{2}}{\partial x_{0} \partial \lambda}
\end{array}\right)+\left(\frac{\partial}{\partial x_{0}} D f\right)\left(\begin{array}{c}
\frac{\partial \varphi_{1}}{\partial \lambda} \\
\frac{\partial \varphi_{2}}{\partial \lambda}
\end{array}\right)+\left(\frac{\partial}{\partial x_{0}} D_{\lambda} f\right) \\
& \frac{d}{d t}\left(\begin{array}{c}
\frac{\partial^{2} \varphi_{1}}{\partial y_{0} \partial \lambda} \\
\frac{\partial^{2} \varphi_{2}}{\partial y_{0} \partial \lambda}
\end{array}\right)=D f\left(\begin{array}{c}
\frac{\partial^{2} \varphi_{1}}{\partial y_{0} \partial \lambda} \\
\frac{\partial^{2} \varphi_{2}}{\partial y_{0} \partial \lambda}
\end{array}\right)+\left(\frac{\partial}{\partial y_{0}} D f\right)\left(\begin{array}{c}
\frac{\partial \varphi_{1}}{\partial \lambda} \\
\frac{\partial \varphi_{2}}{\partial \lambda}
\end{array}\right)+\left(\frac{\partial}{\partial y_{0}} D_{\lambda} f\right)
\end{aligned}
$$

where $D f$ and $D_{\lambda}$ are the derivatives with respect to the state variables $(x, y)$ and the parameter $\lambda$, respectively.

$$
\begin{gathered}
\left(\frac{\partial}{\partial x_{0}} D f\right)=\left(\begin{array}{cc}
\frac{\partial^{2} f_{1}}{\partial x^{2}} \frac{\partial \varphi_{1}}{\partial x_{0}}+\frac{\partial^{2} f_{1}}{\partial y \partial x} \frac{\partial \varphi_{2}}{\partial x_{0}} & \frac{\partial^{2} f_{1}}{\partial x \partial y} \frac{\partial \varphi_{1}}{\partial x_{0}}+\frac{\partial^{2} f_{1}}{\partial y^{2}} \frac{\partial \varphi_{2}}{\partial x_{0}} \\
\frac{\partial^{2} f_{2}}{\partial x^{2}} \frac{\partial \varphi_{1}}{\partial x_{0}}+\frac{\partial^{2} f_{2}}{\partial y \partial x} \frac{\partial \varphi_{2}}{\partial x_{0}} & \frac{\partial^{2} f_{2}}{\partial x \partial y} \frac{\partial \varphi_{1}}{\partial x_{0}}+\frac{\partial^{2} f_{2}}{\partial y^{2}} \frac{\partial \varphi_{2}}{\partial x_{0}}
\end{array}\right) \\
\left(\frac{\partial}{\partial y_{0}} D f\right)=\left(\begin{array}{cc}
\frac{\partial^{2} f_{1}}{\partial x^{2}} \frac{\partial \varphi_{1}}{\partial y_{0}}+\frac{\partial^{2} f_{1}}{\partial y \partial x} \frac{\partial \varphi_{2}}{\partial y_{0}} & \frac{\partial^{2} f_{1}}{\partial x \partial y} \frac{\partial \varphi_{1}}{\partial y_{0}}+\frac{\partial^{2} f_{1}}{\partial y^{2}} \frac{\partial \varphi_{2}}{\partial y_{0}} \\
\frac{\partial^{2} f_{2}}{\partial x^{2}} \frac{\partial \varphi_{1}}{\partial y_{0}}+\frac{\partial^{2} f_{2}}{\partial y \partial x} \frac{\partial \varphi_{2}}{\partial y_{0}} & \frac{\partial^{2} f_{2}}{\partial x \partial y} \frac{\partial \varphi_{1}}{\partial y_{0}}+\frac{\partial^{2} f_{2}}{\partial y^{2}} \frac{\partial \varphi_{2}}{\partial y_{0}}
\end{array}\right) \\
\left(\frac{\partial}{\partial x_{0}} D_{\lambda} f\right)=\left(\begin{array}{c}
\frac{\partial^{2} f_{1}}{\partial x \partial \lambda} \frac{\partial \varphi_{1}}{\partial x_{0}}+\frac{\partial^{2} f_{1}}{\partial y \partial \lambda} \frac{\partial \varphi_{2}}{\partial x_{0}} \\
\frac{\partial^{2} f_{2}}{\partial x \partial \lambda} \frac{\partial \varphi_{1}}{\partial x_{0}}+\frac{\partial^{2} f_{2}}{\partial y \partial \lambda} \frac{\partial \varphi_{2}}{\partial x_{0}}
\end{array}\right),\left(\frac{\partial}{\partial y_{1}} D_{\lambda} f\right)=\left(\begin{array}{c}
\frac{\partial^{2} f_{1}}{\partial y_{0}}+\frac{\partial \varphi_{2}}{\partial y \partial \lambda} \\
\frac{\partial^{2} f_{2}}{\partial x \partial \lambda} \frac{\partial \varphi_{1}}{\partial y_{0}}+\frac{\partial^{2} f_{2}}{\partial y \partial \lambda} \frac{\partial \varphi_{2}}{\partial y_{0}}
\end{array}\right)
\end{gathered}
$$




\section{References}

[1] S.H. Strogatz, Nonlinear Dynamics And Chaos: With Applications To Physics, Biology, Chemistry, And Engineering, Cambridge, Mass., 1994.

[2] http://indy.cs.concordia.ca/auto/

[3] http://www.matcont.ugent.be/

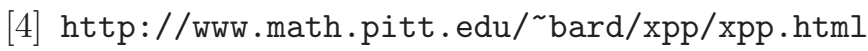

[5] H. Kawakami, "Bifurcation of periodic responses in forced dynamic nonlinear circuits: Computation of bifurcation values of the system parameters," IEEE Trans. Circuits and Systems, vol. 31, no. 3, pp. 248-260, March 1984.

[6] Y. Katsuta and H. Kawakami, "Bifurcations of equilibriums and periodic solutions in nonlinear autonomous system with symmetry," IEICE Trans. Fundam. Electron. Commun. Comput. Sci., vol. J75, no. 6, pp. 1035-1044, June 1992. (in Japanese)

[7] Y. Katsuta and H. Kawakami, "Bifurcations of periodic solutions in nonlinear nonautonomous system with symmetry," IEICE Trans. Fundam. Electron. Commun. Comput. Sci., vol. J76, no. 12, pp. 1753-1760, December 1993. (in Japanese)

[8] T. Yoshinaga and H. Kawakami, "Codimension two bifurcation problems in forced nonlinear circuits," IEICE Trans. Fundam. Electron. Commun. Comput. Sci., vol. 73, no. 6, pp. 817-824, June 1990.

[9] T. Yoshinaga and H. Kawakami, "Bifurcation and chaotic state in forced oscillatory circuits containing saturable inductors," in Nonlinear Dynamics in Circuits, eds. L. Pecora and T. Carroll, pp. 89-119, World Scientific, Singapore, 1995.

[10] H. Kawakami and T. Yoshinaga, "Codimension two bifurcation and its computational algorithm," in Bifurcation and Chaos: Theory and Applications, ed. J. Awrejcewicz, pp. 97-132, Springer Verlag, Berlin, Heidelberg, 1995.

[11] H. Kitajima and H. Kawakami, "An algorithm tracing out the tangent bifurcation curves and its application to Duffing's equation," IEICE Trans. Fundam. Electron. Commun. Comput. Sci., vol. J78, no. 7, pp. 806-810, July 1995. (in Japanese)

[12] H. Kitajima and H. Kawakami, "An algorithm for tracing out the fixed point manifold," IEICE Trans. Fundam. Electron. Commun. Comput. Sci., vol. J79A, no. 5, pp. 1122-1124, May 1996.

[13] T. Ueta, M. Tsueike, H. Kawakami, T. Yoshinaga, and Y. Katsuta, "A computation of bifurcation parameter values for limit cycles," IEICE Trans. Fundam. Electron. Commun. Comput. Sci., vol. E80, no. 9, pp. 1725-1728, September 1997.

[14] T. Kousaka, T. Ueta, and H. Kawakami, "Bifurcation of switched nonlinear dynamical systems," IEEE Trans. Circuits and Systems II, vol. 2, no. 7, pp. 878-885, July 1999.

[15] T. Yoshinaga, Y. Sano, and H. Kawakami, "A method to calculate bifurcations in synaptically coupled Hodgkin-Huxley equations," Int. J. Bifurcation and Chaos, vol. 9, no. 7, pp. 1451-1458, 1999.

[16] T.Ueta and H. Kawakami, "Numerical approaches to bifurcation analysis," in Chaos in circuits and systems, eds. G. Chen and T. Ueta, pp. 593-610, World Scientific, Singapore, 2002.

[17] Y.A. Kuznetsov, Elements of applied bifurcation theory, 3rd Edition, Springer Verlag, New York, 2004.

[18] E.J. Doedel and J.P. Kernévez, AUTO: Software for continuation and bifurcation problems in ordinary differential equations, Applied Mathematics Report, California Institute of Technology, Pasadena, 1986.

[19] T.S. Parker and L.O. Chua, Practical numerical algorithms for chaotic systems, Springer Verlag, Berlin, 1989.

[20] N.E. Nusse, J.A. Yorke, Dynamics: numerical explorations, Springer Verlag, New York, 1994.

[21] T. Matsumoto, M. Komuro, H. Kokubu, and R. Tokunaga, Bifurcations: sights, sounds and mathematics, Springer Verlag, Tokyo, 1993.

[22] K. Shiraiwa, "A generalization of the levinson-massera's equalities," Nagoya Math. J., vol. 67, pp. 121-1458, 1977. 
[23] N. Levinson, "Transformation theory of non-linear differential equations of the second order," Ann. Math., vol. 45, no. 4, pp. 723-737, October 1944.

[24] V.I. Arnold, Geometrical method in the theory of ordinary differential equations, Springer Verlag, New York, 1983.

[25] J. Guckenheimer and P. Holmes, Nonlinear Oscillations, Dynamical Systems, and Bifurcations of Vector Fields, Springer Verlag, New York, 1983.

[26] J.K. Hale and H. Koçak, Dynamics and Bifurcation, Springer Verlag, New York, 1991.

[27] L.S. Pontryagin, Ordinary Differential Equations, Addison-Wesley, Reading, MA, 1962.

[28] T. Ueta, G. Chen, T. Yoshinaga, and H. Kawakami, "A numerical algorithm for computing Neimark-Sacker bifurcation parameters," Proc. ISCAS'99, pp. 503-506, May 1999.

[29] T. Ueta, T. Yoshinaga, H. Kawakami, and G. Chen, "A method to calculate Neimark-Sacker bifurcation in autonomous systems," IEICE Trans. Fundam. Electron. Commun. Comput. Sci., vol. J83A, no. 10, pp. 1141-1147, October 2000. (in Japanese)

[30] T. Yoshinaga and H. Kawakami, "Bifurcations in a BVP equation with periodic impulsive force," Proc. NOLTA'95, pp. 331-334, 1995.

[31] K. Tsumoto, T. Yoshinaga, H. Iida, H. Kawakami, and K. Aihara, "Bifurcations in a mathematical model for circadian oscillations of clock genes," J. Theor. Biol., vol. 239, pp. 101-22, 2006.

[32] K. Tsumoto, G. Kurosawa, T. Yoshinaga, and K. Aihara, "Modeling light adaptation in circadian clock: Prediction of the response that stabilizes entrainment," PLoS ONE, vol. 6, no. 6, e20880, doi:10.1371/journal.pone.0020880, June 2011.

[33] http://bunki.ait.tokushima-u.ac.jp:50080/ 